\title{
Altered epigenetic regulation in early development associated with schizophrenia
}

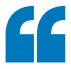

the tumultuous

time around

the age of

onset ... might

be a 'red

herring'
Variations in DNA sites associated with methylation could alter epigenetic regulation during fetal and postnatal neurodevelopment, resulting in an increased risk of schizophrenia, according to two studies published recently in Nature Neuroscience. The findings support the neurodevelopmental hypothesis of schizophrenia and suggest a mechanism through which genetic and environmental risk factors could interact in early neurodevelopment.

DNA methylation is an important form of epigenetic regulation of gene expression. Besides environmental factors, such as diet, smoking and stress, DNA methylation can also be influenced by variations in the DNA sequence at sites referred to as methylation quantitative trait loci (mQTLs). Although mQTLs have been studied in the adult human

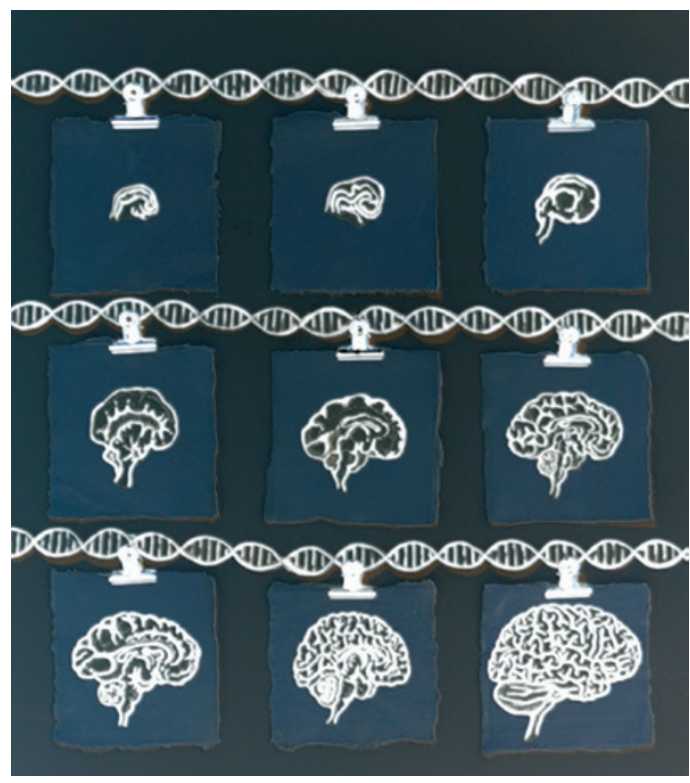

Genetic variation influences DNA methylation, thereby modulating the genome-epigenome interaction that underpins the development of the human brain. Image courtesy of Helen Spiers. brain, little is known about mQTLs in the developing human brain.

Jonathan Mill and colleagues quantified genome-wide patterns of DNA methylation in 166 human fetal brain samples, identifying over 16,000 mQTLs. According to Mill, the majority of the detected mQTLs were cis-acting, but there were also some notable trans-acting mQTLs, indicating long-range interactions in the genome. Mill hopes that the findings will aid understanding of which genomic regions are associated with neurodevelopment disorders.

Interestingly, Mill and colleagues found that fetal brain mQTLs were enriched amongst schizophrenia risk loci identified in a recent large-scale genome-wide association study (GWAS). This finding seems to fit with the prevailing hypothesis that the aetiology of schizophrenia involves a neurodevelopmental component: disruption of brain development in early life could manifest as severe psychiatric symptoms in adulthood.

In another study, Andrew Jaffe and colleagues investigated DNA methylation in schizophrenia across the lifespan. "One of our important research aims was to better define potential epigenetic mechanisms underlying the neurodevelopmental hypothesis of schizophrenia by contrasting changes in DNA methylation across brain development and subsequent dysregulation in schizophrenia."

The researchers evaluated DNA methylation levels at approximately 450,000 sites across the genome in 191 adult patients with schizophrenia and in 335 nonpsychiatric controls over the lifespan.

In line with the study by Mill and colleagues, Jaffe and co-investigators discovered that a substantial proportion of the genetic variants that confer risk for schizophrenia were associated with DNA methylation. "The findings suggest potential mechanisms for epigenetic regulation: these GWAS loci could principally indicate genomic hotspots for sensitivity to the environment through DNA methylation," explains Jaffe.

Moreover, the researchers found that the vast majority of the schizophrenia-associated DNA methylation sites were linked to changes associated with the transition from fetal to postnatal life, rather than corresponding to age-related changes occurring at adolescence or young adulthood, the typical age of onset of schizophrenia. According to Jaffe, the observation implies that both genetic and environmental risk factors for schizophrenia exert their principal effects during fetal development. "The finding suggests that the tumultuous time around the age of onset of schizophrenia might be a 'red herring' in terms of primary pathophysiology," Jaffe contends.

Next, the researchers plan to perform a more thorough molecular characterization of the brain samples from patients with schizophrenia and healthy controls so as to better understand the aetiology of schizophrenia and attempt to identify novel drug targets.

Hemi Malkki

ORIGINAL ARTICLES Jaffe, A. E. et al. Mapping DNA methylation across development, genotype and schizophrenia in the human frontal cortex. Nat. Neurosci. http://dx.doi.org/10.1038/nn.4181 | Hannon, E. et al. Methylation QTLs in the developing brain and their enrichment in schizophrenia loci. Nat. Neurosci. http://dx.doi.org/10.1038/nn.4182 LINKS TO WEBSITES Helen Spiers, one of the study authors, is also an artist. She has an online portfolio at http://helenspiers.com 


\section{ONLINE ONLY}

\section{Highlight Position and Type: lead}

\section{Subject categories:}

Health sciences / Diseases / Psychiatric disorders / Schizophrenia [URI /692/699/476/1799]

Biological sciences / Molecular biology / Epigenetics / DNA methylation [URI /631/337/176/1988]

Health sciences / Diseases / Neurological disorders /

Neurodevelopmental disorders [URI /692/699/375/366]

\section{Techniques terms:}

Biological sciences / Biological techniques / Epigenetics analysis /

Methylation analysis [URI /631/1647/2210/2213]

Biological sciences / Biological techniques / Genomic analysis /

Genome-wide association studies [URI /631/1647/2217/2138] 